\title{
Magnetic circular dichroism versus orbital magnetization
}

\author{
Raffaele Resta ${ }^{*}$ \\ Istituto Officina dei Materiali IOM-CNR, Strada Costiera 11, 34151 Trieste, Italy \\ and Donostia International Physics Center, 20018 San Sebastián, Spain
}

(Received 13 January 2020; accepted 13 April 2020; published 8 May 2020)

\begin{abstract}
The magnetic circular dichroism sum rule yields an extremely useful ground-state observable, which provides a quantitative measure of spontaneous time-reversal symmetry breaking (T-breaking) in a given material. Here I derive its explicit expression within band structure theory, in the general case: trivial insulators, topological insulators, and metals. Orbital magnetization provides a different measure of T-breaking in the electronic ground state. The two observables belong to the class of "geometrical" observables; both are local and admit a "density" in coordinate space. In both of them, one could include or exclude selected groups of bands in order to acquire element-specific information about the T-breaking material. Only in the case of an isolated flat band do the contributions to the two observables coincide. Finally, I provide the corresponding geometrical formula-in a different Hilbert space—-for a many-body interacting system.
\end{abstract}

DOI: 10.1103/PhysRevResearch.2.023139

\section{INTRODUCTION}

Since the very popular 1992 paper by Thole et al. [1], magnetic circular dichroism (MCD) has been widely regarded as an approximate probe of orbital magnetization in bulk solids. Some years later, it was clearly recognized that the MCD sum rule $I_{\alpha \beta}$ (defined below) provides insight into the magnetic properties of solids, although such "magnetic properties" do not coincide with orbital magnetization except in the extreme atomic limit [2]. It must be mentioned that at the time, no sound theory of orbital magnetization in bulk solids was available. Orbital magnetization $\mathbf{M}$ is, by definition, the derivative of the free-energy density with respect to magnetic field (orbital term thereof, and with a minus sign). After Ref. [2], it is then pretty clear that $I_{\alpha \beta}$ is the free-energy derivative with respect to a different Tbreaking probe: circularly polarized light, integrated over the whole spectrum. Owing to a fluctuation-dissipation theorem, a frequency-integrated dynamical probe becomes effectively a static one; the said probe has the virtue of coupling to orbital degrees of freedom only. The two observables $\mathbf{M}$ and $I_{\alpha \beta}$ provide two quantitatively different measures of spontaneous T-breaking in the orbital degrees of freedom of a given material. On the experimental side, $I_{\alpha \beta}$ is naturally endowed with core chemical specificity, at the root of its great success; while instead only the total $\mathbf{M}$ value is experimentally accessible.

Condensed-matter physics adopts Born-von-Kàrmàn periodic boundary conditions (PBCs), at the root of Bloch's theorem, which in turn allows one to address the intensive

\footnotetext{
*resta@iom.cnr.it

Published by the American Physical Society under the terms of the Creative Commons Attribution 4.0 International license. Further distribution of this work must maintain attribution to the author(s) and the published article's title, journal citation, and DOI.
}

electronic properties of crystalline materials. Unfortunately, PBCs are incompatible with the presence of a generic magnetic field: for these reasons, the theory of orbital magnetization was only established in 2006 [3,4]. Therein, $\mathbf{M}$ is expressed as a reciprocal-space integral; it is worth stressing that no use is made of the angular momentum $L_{z}$, a "forbidden" operator within PBCs. The operator $L_{z}$ is only legitimate when addressing a bounded crystallite.

Here, I thoroughly investigate the analogies and differences between the two observables, also providing three significant advances. (i) I give a microscopic expression for $I_{\alpha \beta}$, gauge invariant in form, for any crystalline material (either metal or insulator) within band structure theory. I also show that in a Chern insulator, $I_{\alpha \beta}$ is not affected by the topologically protected edge states (while $\mathbf{M}$ is affected). (ii) I show that $I_{\alpha \beta}$ is a local observable, in full analogy to $\mathbf{M}[5,6]$. It must be stressed that other geometrical observables are strongly nonlocal (most notably electrical polarization [5]). (iii) I go beyond band structure theory and I show that even in a correlated many-electron system, $I_{\alpha \beta}$ is a geometrical ground-state observable, although in a different Hilbert space. When PBCs are abandoned, the very same geometrical formula for $I_{\alpha \beta}$ goes seamlessly into the center-of-mass angular momentum formula of Ref. [2] (which only makes sense for a bounded crystallite).

\section{THEORY}

Shortly after the theory of orbital magnetization was fully established [3], Souza and Vanderbilt derived an explicit expression for $I_{\alpha \beta}$ in the special case of a topologically trivial insulator [7]. It was shown that both $\mathbf{M}$ and $I_{\alpha \beta}$ are geometrical properties of the electronic ground state; an explicit expression for their difference was also provided.

The MCD sum rule concerns the frequency integral of the imaginary part of the antisymmetric term in the conductivity 
tensor,

$$
I_{\alpha \beta}=\operatorname{Im} \int_{0}^{\infty} d \omega \sigma_{\alpha \beta}^{(-)}(\omega) ;
$$

a kind of fluctuation-dissipation theorem relates $I_{\alpha \beta}$ to a ground-state property, both for a bounded sample (e.g., a crystallite) within the so-called open boundary conditions (OBCs), and for an unbounded solid within PBCs. In both frameworks, all ground-state properties-at the independentparticle level - can be expressed in terms of the relevant ground-state projector $\mathcal{P}$.

In the OBCs case, the projector (per spin channel) is

$$
\mathcal{P}=\sum_{\epsilon_{j} \leqslant \mu}\left|\varphi_{j}\right\rangle\left\langle\varphi_{j}\right|,
$$

where $\mu$ is the Fermi level and $\left|\varphi_{j}\right\rangle$ are the single-particle eigenstates of the Hamiltonian $\mathcal{H}$ with eigenvalues $\epsilon_{j}$. In the band structure case, the projector is instead

$$
\mathcal{P}=V_{\text {cell }} \int_{\mathrm{BZ}} \frac{d \mathbf{k}}{(2 \pi)^{d}} \sum_{\epsilon_{j \mathbf{k}} \leqslant \mu}\left|\psi_{j \mathbf{k}}\right\rangle\left\langle\psi_{j \mathbf{k}}\right|,
$$

where $\mathrm{BZ}$ is the Brillouin zone, $\left|\psi_{j \mathbf{k}}\right\rangle$ are the Bloch orbitals normalized to one in the crystal cell of volume $V_{\text {cell }}, \epsilon_{j \mathbf{k}}$ are the band energies, and $d$ is the dimension; $V_{\text {cell }}$ must be understood as the area for $d=2$. The reason for adopting the same symbol $\mathcal{P}$ in Eqs. (2) and (3) lies in the "nearsightedness" principle [8]. If one evaluates $\mathcal{P}$ from Eq. (2) for a large bounded crystallite, and then further projects this $\mathcal{P}$ onto the inner region of the crystallite, the result asymptotically converges to the $\mathcal{P}$ value provided by Eq. (3) for the same material; the convergence is exponential in insulators and power law in metals.

We start with a bounded sample within OBCs: the sum rule for $I_{\alpha \beta}$ has a relatively straightforward expression [7]. A tedious calculation (see the Appendix) shows that it can be equivalently expressed as

$$
I_{\alpha \beta}=-\frac{i \pi e^{2}}{2 \hbar^{2} V} \operatorname{Tr}\left\{(\mathcal{H}-\mu)\left[\left[r_{\alpha}, \mathcal{P}\right],\left[r_{\beta}, \mathcal{P}\right]\right]\right\} .
$$

The virtue of this expression becomes clear when switching to $\mathrm{PBCs}$ and band structure theory. In the latter case, $\mathcal{P}$ is lattice periodical,

$$
\left\langle\mathbf{r}|\mathcal{P}| \mathbf{r}^{\prime}\right\rangle=\left\langle\mathbf{r}+\mathbf{R}|\mathcal{P}| \mathbf{r}^{\prime}+\mathbf{R}\right\rangle,
$$

where $\mathbf{R}$ is a lattice vector. The position $\mathbf{r}$ is instead a forbidden operator, incompatible with PBCs [9]. Notwithstanding, the commutator $[\mathbf{r}, \mathcal{P}]$ is an honest lattice-periodical operator (like $\mathcal{H}$ and $\mathcal{P}$ ), and hence Eq. (4) can be adopted as it is,

$$
I_{\alpha \beta}=-\frac{i \pi e^{2}}{2 \hbar^{2} V_{\text {cell }}} \operatorname{Tr}_{\text {cell }}\left\{(\mathcal{H}-\mu)\left[\left[r_{\alpha}, \mathcal{P}\right],\left[r_{\beta}, \mathcal{P}\right]\right]\right\} .
$$

Therein, $\mathcal{H}$ is the periodic Hamiltonian and $\mathcal{P}$ is given by Eq. (3).

The next step is to express Eq. (6) as a Fermi volume integral (BZ integral in the insulating case), as customary for any intensive ground-state observable; its geometrical nature will also perspicuously appear. We cast Eq. (3) in terms of the periodic Bloch orbitals $\left|u_{j \mathbf{k}}\right\rangle=e^{-i \mathbf{k} \cdot \mathbf{r}}\left|\psi_{j \mathbf{k}}\right\rangle$, eigenvectors of $\mathcal{H}_{\mathbf{k}}=e^{-i \mathbf{k} \cdot \mathbf{r}} \mathcal{H} e^{i \mathbf{k} \cdot \mathbf{r}}$ :

$$
\begin{aligned}
\left\langle\mathbf{r}|\mathcal{P}| \mathbf{r}^{\prime}\right\rangle= & V_{\text {cell }} \int_{\mathrm{BZ}} \frac{d \mathbf{k}}{(2 \pi)^{d}} e^{i \mathbf{k} \cdot\left(\mathbf{r}-\mathbf{r}^{\prime}\right)}\left\langle\mathbf{r}\left|\mathcal{P}_{\mathbf{k}}\right| \mathbf{r}^{\prime}\right\rangle, \\
& \mathcal{P}_{\mathbf{k}}=\sum_{\epsilon_{j \mathbf{k}} \leqslant \mu}\left|u_{j \mathbf{k}}\right\rangle\left\langle u_{j \mathbf{k}}\right| .
\end{aligned}
$$

In order to establish a differential geometry in the space of the $\left|u_{j \mathbf{k}}\right\rangle$ state vectors, we choose a gauge which makes the $\left|u_{j \mathbf{k}}\right\rangle$ smooth (i.e., $C^{\infty}$ ) throughout the whole BZ. This is always possible, even in topologically nontrivial materials [10]. The integrand in Eq. (7) is periodical in $\mathbf{k}$, and hence the $\mathrm{BZ}$ integral of its $\mathbf{k}$ derivative vanishes:

$0=i\left(\mathbf{r}-\mathbf{r}^{\prime}\right)\left\langle\mathbf{r}|\mathcal{P}| \mathbf{r}^{\prime}\right\rangle+V_{\text {cell }} \int_{\mathrm{BZ}} \frac{d \mathbf{k}}{(2 \pi)^{d}} e^{i \mathbf{k} \cdot\left(\mathbf{r}-\mathbf{r}^{\prime}\right)}\left\langle\mathbf{r}\left|\partial_{\mathbf{k}} \mathcal{P}_{\mathbf{k}}\right| \mathbf{r}^{\prime}\right\rangle$,

$$
i[\mathbf{r}, \mathcal{P}]=-V_{\text {cell }} \int_{\mathrm{BZ}} \frac{d \mathbf{k}}{(2 \pi)^{d}} e^{i \mathbf{k} \cdot \mathbf{r}} \partial_{\mathbf{k}} \mathcal{P}_{\mathbf{k}} e^{-i \mathbf{k} \cdot \mathbf{r}} .
$$

We are now ready to replace this into Eq. (6), together with

$$
\mathcal{H}=V_{\text {cell }} \int_{\mathrm{BZ}} \frac{d \mathbf{k}}{(2 \pi)^{d}} e^{i \mathbf{k} \cdot \mathbf{r}} \mathcal{H}_{\mathbf{k}} e^{-i \mathbf{k} \cdot \mathbf{r}} .
$$

The three reciprocal-space integrals in the product contract to one (see the Appendix), and we arrive at

$$
I_{\alpha \beta}=\frac{i \pi e^{2}}{2 \hbar^{2}} \int_{\mathrm{BZ}} \frac{d \mathbf{k}}{(2 \pi)^{d}} \operatorname{Tr}_{\text {cell }}\left\{\left(\mathcal{H}_{\mathbf{k}}-\mu\right)\left[\partial_{k_{\alpha}} \mathcal{P}_{\mathbf{k}}, \partial_{k_{\beta}} \mathcal{P}_{\mathbf{k}}\right]\right\} .
$$

The sum rule $I_{\alpha \beta}$, defined in Eq. (1), is the frequency integral of a linear response function; Eq. (12) shows that it coincides with a ground-state material property. Equalities of this kind belong to the general class of fluctuation-dissipation theorems [7].

Equation (12) is one of the major results of the present work. It applies on the same ground to trivial insulators, topological insulators, and metals. In the metallic case, the k derivative of $\mathcal{P}_{\mathbf{k}}$ includes a $\delta$-like singularity at the Fermi level, which is annihilated by antisymmetrization. When specialized to the so-called Hamiltonian gauge [4], Eq. (12) yields the same formula as in Ref. [7] (derived therein in a completely different way for trivial insulators only).

Intensive material properties in crystalline materials are expressed as reciprocal-space integrals, such as $I_{\alpha \beta}$ in Eq. (12); the analogous expression for orbital magnetization $\mathbf{M}$ was found in 2006 [3,4]. While the observables have obviously different dimensions, the two reciprocal-space integrals are similar-when expressed within the present formalism-but not equal. Preliminarily, we observe that custom dictates the adoption of the field $\mathbf{H}$ in the free energy. Instead, because of the reasons well explained in Ref. [11], first-principles theory alternatively adopts the more fundamental field $\mathbf{B}$. The (by now classic) orbital magnetization expression, formulated in the Hamiltonian gauge $[3,4]$, can be shown to be equivalent to the gauge-invariant expression [12]

$$
\mathbf{M}=\frac{i e}{2 \hbar c} \int_{\mathrm{BZ}} \frac{d \mathbf{k}}{(2 \pi)^{d}} \operatorname{Tr}_{\text {cell }}\left\{\left|\mathcal{H}_{\mathbf{k}}-\mu\right|\left(\partial_{\mathbf{k}} \mathcal{P}_{\mathbf{k}}\right) \times\left(\partial_{\mathbf{k}} \mathcal{P}_{\mathbf{k}}\right)\right\},
$$


where $\left|\mathcal{H}_{\mathbf{k}}-\mu\right|=\left(\mathcal{H}_{\mathbf{k}}-\mu\right)\left(\mathcal{I}-2 \mathcal{P}_{\mathbf{k}}\right)$ is the operator which acts as $\left(\mu-\mathcal{H}_{k}\right)$ on the occupied states, and as $\left(\mathcal{H}_{\mathbf{k}}-\mu\right)$ on the unoccupied ones. Comparing to the reciprocal-space expression of the MCD sum rule, given by Eq. (12), the key difference is the presence or absence of the modulus of the operator $\left(\mathcal{H}_{\mathbf{k}}-\mu\right)$ (besides the obvious prefactor).

We stress that $\mathcal{P}_{\mathbf{k}}$ is gauge invariant in the generalized sense, i.e., by arbitrary mixing of the occupied $\left|u_{j \mathbf{k}}\right\rangle$ orbitals at a given k [4]. Therefore, Eqs. (12) and (13) are gauge invariant in form. Their geometrical integrands are simple modifications of the Berry curvature, whose known expression is

$$
\Omega_{\alpha \beta}(\mathbf{k})=i \operatorname{Tr}_{\text {cell }}\left\{\mathcal{P}_{\mathbf{k}}\left[\partial_{k_{\alpha}} \mathcal{P}_{\mathbf{k}}, \partial_{k_{\beta}} \mathcal{P}_{\mathbf{k}}\right]\right\} \text {. }
$$

Therein, the band projector $\mathcal{P}_{\mathbf{k}}$ is the sole ingredient, while the Hamiltonian enters Eqs. (12) and (13) as well. It enters both formulas via $\left(\mathcal{H}_{\mathbf{k}}-\mu\right)$ for aesthetical reasons, i.e., to explicitly display their invariance by translation of the energy zero. Nonetheless, the $\mu$ dependence can be dropped in $I_{\alpha \beta}$ since the $\mu$-dependent term is annihilated after antisymmetrization. In this regard, $\mathbf{M}$ is different: it is $\mu$ independent in trivial insulators, while it actually depends on $\mu$ (in the gap) in Chern insulators [13]. Remarkably, this means that-in a bounded sample - the topologically protected boundary states contribute to $\mathbf{M}$, but not to $I_{\alpha \beta}$.

Finally, I point out another major appealing feature of Eqs. (6) and (12): both show the locality of the observable. If we define the (real) lattice-periodical function $\mathcal{X}_{\alpha \beta}(\mathbf{r})$ as

$$
\mathcal{X}_{\alpha \beta}(\mathbf{r})=\frac{\pi e^{2}}{2 \hbar^{2}} \operatorname{Im}\left\langle\mathbf{r}\left|(\mathcal{H}-\mu)\left[\left[r_{\alpha}, \mathcal{P}\right],\left[r_{\beta}, \mathcal{P}\right]\right]\right| \mathbf{r}\right\rangle,
$$

the sum rule reads

$$
I_{\alpha \beta}=\frac{1}{V_{\text {cell }}} \int_{\text {cell }} d \mathbf{r} \mathcal{X}_{\alpha \beta}(\mathbf{r}) .
$$

The function $\mathcal{X}_{\alpha \beta}(\mathbf{r})$ assumes then the meaning of a MCD "density" and provides spatial resolution, although such resolution is not expected to be microscopic, only macroscopic. Previous experience with other local geometrical observables, mostly based on model Hamiltonians, has shown-when dealing, e.g., with a heterostructure - that the "density" separates the contributions from the different regions of the sample [14-16]. Obviously, the k-space formula in the supercell formalism lacks spatial resolution even at the macroscopic level.

The concept of orbital magnetization density has been proposed and validated in Refs. [5,6,13]. Its macroscopic average coincides, by definition, with (minus) the $\mathbf{B}$ derivative of the orbital free-energy density (spin magnetization is obviously local as well). As said in Sec. I, the apparently analogous case of electrical polarization is instead strongly nonlocal: no density can be defined $[4,5]$.

\section{CONTRIBUTION FROM AN ISOLATED FLAT BAND}

Whenever the band spectrum comprises an isolated band, its contribution to both observables can be evaluated by including that band only in the ground-state projector. This parallels the spectral selectivity naturally provided by the sum rule, and largely exploited by MCD experimentalists. The single-band expressions for the two observables are, in the case of full occupancy $[3,4,7]$,

$$
\begin{aligned}
I_{\alpha \beta} & =\frac{\pi e^{2}}{\hbar^{2}} \operatorname{Im} \int_{\mathrm{BZ}} \frac{d \mathbf{k}}{(2 \pi)^{d}}\left\langle\partial_{k_{\alpha}} u_{\mathbf{k}}\left|\left(\mathcal{H}_{\mathbf{k}}-\epsilon_{\mathbf{k}}\right)\right| \partial_{k_{\beta}} u_{\mathbf{k}}\right\rangle, \\
M_{\gamma} & =\frac{e}{2 \hbar c} \varepsilon_{\gamma \alpha \beta} \operatorname{Im} \int_{\mathrm{BZ}} \frac{d \mathbf{k}}{(2 \pi)^{d}}\left\langle\partial_{k_{\alpha}} u_{\mathbf{k}}\left|\left(\mathcal{H}_{\mathbf{k}}+\epsilon_{\mathbf{k}}\right)\right| \partial_{k_{\beta}} u_{\mathbf{k}}\right\rangle,
\end{aligned}
$$

where summation over repeated indices is implicit, and $\varepsilon_{\gamma \alpha \beta}$ is the antisymmetric tensor (in the case of $\mathbf{M}$, the argument of "Im" is purely imaginary after antisymmetrization). For a flat core band, the difference between the two integrals is a constant times the Chern invariant, which in turn vanishes by assumption.

We remind the reader that in the general case, the orbital moment per cell as such is an ill-defined quantity: both $\mathbf{M}$ and $I_{\alpha \beta}$ have the primary meaning of a free-energy derivative. Only in the extreme case of an isolated flat band can they both be alternatively expressed as the core orbital moment per cell.

Switching to vector notations, Eq. (18) becomes

$$
\mathbf{M}=-\frac{i e}{2 \hbar c} \int_{\mathrm{BZ}} \frac{d \mathbf{k}}{(2 \pi)^{d}}\left\langle\partial_{\mathbf{k}} u_{\mathbf{k}}\left|\mathcal{H}_{\mathbf{k}} \times\right| \partial_{\mathbf{k}} u_{\mathbf{k}}\right\rangle .
$$

If $\varphi(\mathbf{r})$ is the core orbital in the Schrödinger representation, the zero-hopping assumption yields

$$
\begin{gathered}
\left\langle\mathbf{r} \mid u_{\mathbf{k}}\right\rangle=\sum_{\mathbf{R}} e^{i \mathbf{k} \cdot(\mathbf{R}-\mathbf{r})} \varphi(\mathbf{r}-\mathbf{R}), \\
\left\langle\mathbf{r} \mid \partial_{\mathbf{k}} u_{\mathbf{k}}\right\rangle=i \sum_{\mathbf{R}}(\mathbf{R}-\mathbf{r}) e^{i \mathbf{k} \cdot(\mathbf{R}-\mathbf{r})} \varphi(\mathbf{r}-\mathbf{R}),
\end{gathered}
$$

where $\mathbf{R}$ are the lattice vectors. Exploiting the zero-overlap assumption, and the fact that $\mathcal{H}_{\mathbf{k}}$ commutes with $e^{i \mathbf{k} \cdot \mathbf{R}}$, we get

$$
\begin{aligned}
\left\langle\partial_{\mathbf{k}} u_{\mathbf{k}}\left|\mathcal{H}_{\mathbf{k}} \times\right| \partial_{\mathbf{k}} u_{\mathbf{k}}\right\rangle= & \int_{\text {cell }} d \mathbf{r} \sum_{\mathbf{R}} \varphi^{*}(\mathbf{R}-\mathbf{r}) e^{i \mathbf{k} \cdot \mathbf{r}} \mathcal{H}_{\mathbf{k}} \\
& \times e^{-i \mathbf{k} \cdot \mathbf{r}}(\mathbf{R}-\mathbf{r}) \varphi(\mathbf{r}-\mathbf{R}) .
\end{aligned}
$$

Since $\mathcal{H}_{\mathbf{k}}=e^{-i \mathbf{k} \cdot \mathbf{r}} \mathcal{H} e^{i \mathbf{k} \cdot \mathbf{r}}$, the above expression is $\mathbf{k}$ independent. Then,

$\mathbf{M}=-\frac{i e}{2 \hbar c V_{\text {cell }}}\left\langle\partial_{\mathbf{k}} u_{\mathbf{k}}\left|\mathcal{H}_{\mathbf{k}} \times\right| \partial_{\mathbf{k}} u_{\mathbf{k}}\right\rangle=\frac{i e}{2 \hbar c V_{\text {cell }}}\langle\varphi|\mathcal{H} \times \mathbf{r}| \varphi\rangle$.

Finally, using $\mathbf{r} \times \mathbf{r}=0$ and $\mathbf{v}=i[\mathcal{H}, \mathbf{r}] / \hbar$, we obtain

$$
\mathbf{M}=\frac{i e}{2 \hbar c V_{\text {cell }}}\langle\varphi|[\mathcal{H}, \mathbf{r}] \times \mathbf{r}| \varphi\rangle=-\frac{e}{2 c V_{\text {cell }}}\langle\varphi|\mathbf{r} \times \mathbf{v}| \varphi\rangle,
$$

thus retrieving the magnetic analog of the popular ClausiusMossotti model polarization in electrostatics. Such an expression makes sense only because the electron distribution is confined in disconnected core regions, separated by empty interstitial space; and therefore the unbounded nature of $\mathbf{r}$ does no harm [9].

In most cases-such as for $d$ levels in transition metalsthe band is not flat, not isolated, and/or composite. Nonetheless, most of the MCD literature $[1,17,18]$ assumes that $I_{\alpha \beta}$ provides an approximate measure of orbital magnetization. The identification of the two integrals in Eqs. (17) and (18) 
is not justified, not even approximately: the Berry curvature is known to be a weird function of $\mathbf{k}$ (see, e.g., Fig. 5.8 in Ref. [4]).

\section{CORRELATED GROUND STATE}

At this point, we abandon band structure theory altogether and we switch to dealing with an interacting system. The formula was provided by Kunes and Oppeneer in 2000 [2] for a bounded crystallite (or even a noncrystalline sample); it cannot be adopted within PBCs, given that one of its entries is the position operator $\mathbf{r}$, forbidden within PBCs [9]. Here we provide the many-body formula in terms of a many-body ground state, and we show that even in this case, $I_{\alpha \beta}$ is a geometrical observable, although in a different Hilbert space. For the sake of simplicity, we deal with the simple case where a purely orbital Hamiltonian can be established for each spin channel (same as above for the band structure case).

We consider, following a milestone paper by Kohn [19], a system of $N$ interacting particles in a cubic box of volume $L^{3}$, and the family of many-body Hamiltonians parametrized by the parameter $\boldsymbol{\kappa}$ :

$$
\hat{H}_{\boldsymbol{\kappa}}=\frac{1}{2 m} \sum_{i=1}^{N}\left[\mathbf{p}_{i}+\frac{e}{c} \mathbf{A}\left(\mathbf{r}_{i}\right)+\hbar \boldsymbol{\kappa}\right]^{2}+\hat{V},
$$

where $\hat{V}$ includes one-body and two-body potentials. The vector potential $\mathbf{A}(\mathbf{r})$ summarizes all T-breaking terms, as, e.g., those due to spin-orbit coupling to a background of local moments. We adopt Born-von-Kàrmàn PBCs over each electron coordinate $\mathbf{r}_{i}$ independently, whose Cartesian components $r_{i, \alpha}$ are then equivalent to the angles $2 \pi r_{i, \alpha} / L$. The potential $\hat{V}$ and the vector potential $\mathbf{A}(\mathbf{r})$ enjoy the same periodicity. The vector $\boldsymbol{\kappa}$, having the dimensions of an inverse length, is called "flux" or "twist." Setting $\kappa \neq 0$ amounts to a gauge transformation; since PBCs violate gauge invariance, the eigenvectors $\left|\Psi_{n \kappa}\right\rangle$ and the eigenvalues $E_{n} \boldsymbol{\kappa}$ have a nontrivial $\boldsymbol{\kappa}$ dependence [19].

In order to simplify notations, we define $\hat{H}_{0} \equiv \hat{H},\left|\Psi_{n 0}\right\rangle \equiv$ $\left|\Psi_{n}\right\rangle, E_{n 0} \equiv E_{n}$, and $\omega_{0 n}=\left(E_{n}-E_{0}\right) / \hbar$. If we further define the $\kappa=0$ many-body velocity operator as

$$
\hat{\mathbf{v}}=\frac{1}{m} \sum_{i=1}^{N}\left[\mathbf{p}_{i}+\frac{e}{c} \mathbf{A}\left(\mathbf{r}_{i}\right)\right]=\frac{1}{\hbar} \partial_{\boldsymbol{\kappa}} \hat{H},
$$

the Kubo formula for the imaginary part of the conductivity straightforwardly yields the sum rule:

$$
I_{\alpha \beta}=\frac{\pi e^{2}}{\hbar L^{3}} \operatorname{Im} \sum_{n \neq 0} \frac{\left\langle\Psi_{0}\left|\hat{v}_{\alpha}\right| \Psi_{n}\right\rangle\left\langle\Psi_{n}\left|\hat{v}_{\beta}\right| \Psi_{0}\right\rangle}{\omega_{0 n}} ;
$$

the task is now to convert this expression into a ground-state property.

The many-body analog of the $\mathbf{k} \cdot \mathbf{p}$ expansion yields

$$
\begin{gathered}
\left|\partial_{\boldsymbol{\kappa}} \Psi_{0}\right\rangle=-\sum_{n \neq 0}\left|\Psi_{n}\right\rangle \frac{\left\langle\Psi_{n}|\hat{\mathbf{v}}| \Psi_{0}\right\rangle}{\omega_{n 0}}, \\
\left(\hat{H}-E_{0}\right)\left|\partial_{\boldsymbol{\kappa}} \Psi_{0}\right\rangle=-\sum_{n \neq 0}\left|\Psi_{n}\right\rangle\left\langle\Psi_{n}|\hat{\mathbf{v}}| \Psi_{0}\right\rangle .
\end{gathered}
$$

By comparing these expression to Eq. (27), we find our final geometrical formula,

$$
I_{\alpha \beta}=\frac{\pi e^{2}}{\hbar^{2} L^{3}} \operatorname{Im}\left\langle\partial_{\kappa_{\alpha}} \Psi_{0}\left|\left(\hat{H}-E_{0}\right)\right| \partial_{\kappa_{\beta}} \Psi_{0}\right\rangle .
$$

The correspondence with the single-band formula of Eq. (17) is self-evident; a gauge-invariant expression can be easily obtained from the ground-state projector $\hat{P}_{\boldsymbol{\kappa}}=\left|\Psi_{\boldsymbol{\kappa}}\right\rangle\left\langle\Psi_{\boldsymbol{\kappa}}\right|$. At variance with the band structure case, the many-body formula for $I_{\alpha \beta}$ unfortunately cannot be compared with a corresponding formula for M. To this day, such a formula does not exist: the orbital magnetization of a correlated many-body wave function within PBCs is currently an open (and challenging) problem. The corresponding problem for electrical polarization has been solved long ago [9].

From now on, we adopt the same Kohn Hamiltonian of Eq. (25), but we switch from PBCs to OBCs, thus addressing a crystallite (or any bounded sample). In this case, the flux $\kappa$ can be easily "gauged away": the energies are $\boldsymbol{\kappa}$ independent, while the state vectors are

$$
\left|\Psi_{n \boldsymbol{\kappa}}\right\rangle=e^{-i \boldsymbol{\kappa} \cdot \hat{\mathbf{r}}}\left|\Psi_{n}\right\rangle, \quad \text { OBCs, }
$$

where $\hat{\mathbf{r}}=\sum_{i} \mathbf{r}_{i}$ is the many-body position, a trivial multiplicative operator within OBCs. Therefore,

$$
\begin{gathered}
\left|\partial_{\boldsymbol{\kappa}} \Psi_{0}\right\rangle=-i \hat{\mathbf{r}}\left|\Psi_{0}\right\rangle, \\
I_{\alpha \beta}=\frac{\pi e^{2}}{\hbar^{2} L^{3}} \operatorname{Im}\left\langle\Psi_{0}\left|\hat{r}_{\alpha}\left(\hat{H}-E_{0}\right) \hat{r}_{\beta}\right| \Psi_{0}\right\rangle \\
=-\frac{i \pi e^{2}}{2 \hbar^{2} L^{3}} \operatorname{Im}\left\langle\Psi_{0}\left|\hat{r}_{\alpha}\left[\hat{H}, \hat{r}_{\beta}\right]\right| \Psi_{0}\right\rangle \\
=-\frac{\pi e^{2}}{2 \hbar L^{3}}\left\langle\Psi_{0}\left|\left(\hat{r}_{\alpha} \hat{v}_{\beta}-\hat{r}_{\beta} \hat{v}_{\alpha}\right)\right| \Psi_{0}\right\rangle .
\end{gathered}
$$

Not surprisingly, this is identical to the center-of mass angular momentum formula, first derived in Ref. [2].

By expanding the many-body operators $\hat{\mathbf{r}}$ and $\hat{\mathbf{v}}$, Eq. (33) is seen to be proportional to the ground-state expectation value of $\sum_{i i^{\prime}} \mathbf{r}_{i} \times \mathbf{v}_{i^{\prime}}$, while the orbital moment of a bounded sample is proportional to the ground-state expectation value of $\sum_{i} \mathbf{r}_{i} \times \mathbf{v}_{i}$. The two coincide only in the single-electron case, consistently with Eq. (24) above: the case of an isolated flat band amounts, in fact, to considering one electron per cell (and per spin channel).

\section{CONCLUSIONS}

I have presented a microscopic expression for the MCD sum rule $I_{\alpha \beta}$ within band structure theory and compared it to the analogous formula for orbital magnetization M. Both geometrical formulas are gauge invariant in form and very general: they address trivial insulators, topological insulators, and metals on the same footing. In a Chern insulator, the topologically protected edge states contribute to M. I prove here that they do not contribute to $I_{\alpha \beta}$. I have also proved the locality of $I_{\alpha \beta}$, i.e., its spatial-resolution capability (M is known to be local [5,6]). In agreement with common wisdom, the present formulation confirms that the contributions to $I_{\alpha \beta}$ and to $\mathbf{M}$ by an isolated flat band of nonoverlapping core states coincide, and also coincide with the 
intuitive-Clausius-Mossotti inspired-definition of $\mathbf{M}$ as the core orbital moment over the cell volume. I have also presented the many-body version of $I_{\alpha \beta}$, which is a geometrical ground-state observable as well, although in a different Hilbert space.

It is difficult to say anything about to which extent the two observables could provide similar results in some classes of materials (and perhaps very different in others), for the good reason that few first-principles calculations of $\mathbf{M}$ exist [20,21], and none of the sum rule (to the best of my knowledge). A further drawback is that "first-principles" is regarded as a synonym of density functional theory, whose accuracy in addressing T-breaking properties and materials is known to be poor [20].

With respect to magnetization measurements, the MCD sum rule $I_{\alpha \beta}$ has two great virtues, at the root of its enormous success. The first one is that $I_{\alpha \beta}$ is a purely orbital property, while in magnetization measurements the spin contribution has to be subtracted; further complications arise from shape issues and demagnetization coefficients. The second major virtue is that the frequency integral can be (and routinely is) performed over selected spectral ranges, thus isolating the T-breaking contributions from selected orbital shells, and providing element- and/or site-specific information. The MCD sum rule $I_{\alpha \beta}$ is an outstanding ground-state observable per se; there is no compelling reason for identifying it (even approximately) with some form of orbital magnetization.

\section{ACKNOWLEDGMENTS}

Useful discussions with M. Altarelli and I. Souza are gratefully acknowledged. This work is supported by the ONR Grant No. N00014-17-1-2803.

\section{APPENDIX}

\section{MCD sum rule in a bounded sample}

Besides $\mathcal{P}$, here we need its complementary $\mathcal{Q}=\mathcal{I}-\mathcal{P}$,

$$
\mathcal{Q}=\sum_{\epsilon_{j}>\mu}\left|\varphi_{j}\right\rangle\left\langle\varphi_{j}\right| .
$$

Following Ref. [7], the MCD sum rule reads

$$
\begin{aligned}
I_{\alpha \beta} & =\frac{\pi e^{2}}{2 \hbar V} \operatorname{Tr}\left\{\mathcal{P} r_{\alpha} \mathcal{Q} v_{\beta}\right\}-(\alpha \leftrightarrow \beta) \\
& \doteq \frac{\pi e^{2}}{2 \hbar V} \operatorname{Tr}\left\{\mathcal{P} r_{\alpha} \mathcal{Q} v_{\beta}\right\} \\
& \doteq \frac{i \pi e^{2}}{2 \hbar^{2} V} \operatorname{Tr}\left\{\mathcal{P} r_{\alpha} \mathcal{Q}\left[\mathcal{H}, r_{\beta}\right]\right\},
\end{aligned}
$$

where the symbol $\doteq$ means "equal after antisymmetrization", and $v_{\beta}=i\left[\mathcal{H}, r_{\beta}\right] / \hbar$ has been used. We then switch to a $\mathcal{P}$ only formula:

$$
\begin{aligned}
\operatorname{Tr}\left\{\mathcal{P} r_{\alpha} \mathcal{Q}\left[\mathcal{H}, r_{\beta}\right]\right\} \doteq & \operatorname{Tr}\left\{\mathcal{P} r_{\alpha} \mathcal{Q H} r_{\beta}\right\}-\operatorname{Tr}\left\{\mathcal{P} r_{\alpha} \mathcal{Q} r_{\beta} \mathcal{H}\right\} \\
\doteq & \operatorname{Tr}\left\{\mathcal{P} r_{\alpha} \mathcal{H} r_{\beta}\right\}-\operatorname{Tr}\left\{\mathcal{P} r_{\alpha} \mathcal{P} \mathcal{H} r_{\beta}\right\} \\
& +\operatorname{Tr}\left\{\mathcal{P} r_{\alpha} \mathcal{P} r_{\beta} \mathcal{H}\right\},
\end{aligned}
$$

where a symmetric term has been dropped. Using then the cyclic invariance of the trace and the fact that $\mathcal{H}$ commutes with $\mathcal{P}$, Eq. (A3) is recast as

$$
\begin{aligned}
\operatorname{Tr}\left\{\mathcal{P} r_{\alpha} \mathcal{Q}\left[\mathcal{H}, r_{\beta}\right]\right\} \doteq & \operatorname{Tr}\left\{\mathcal{H} r_{\beta} \mathcal{P} r_{\alpha}\right\}-\operatorname{Tr}\left\{\mathcal{H} \mathcal{P} r_{\beta} \mathcal{P} r_{\alpha}\right\} \\
& +\operatorname{Tr}\left\{\mathcal{H} \mathcal{P} r_{\alpha} \mathcal{P} r_{\beta}\right\} \\
\doteq & \operatorname{Tr}\left\{\mathcal{H}(2 \mathcal{P}-\mathcal{I}) r_{\alpha} \mathcal{P} r_{\beta}\right\}
\end{aligned}
$$

It is then easy to verify that

$$
\operatorname{Tr}\left\{\mathcal{H}(2 \mathcal{P}-\mathcal{I}) r_{\alpha} \mathcal{P} r_{\beta}\right\} \doteq-\operatorname{Tr}\left\{\mathcal{H}\left[r_{\alpha}, \mathcal{P}\right]\left[r_{\beta}, \mathcal{P}\right]\right\},
$$

where again a symmetric term has been dropped.

We thus arrive at

$$
I_{\alpha \beta}=-\frac{i \pi e^{2}}{2 \hbar^{2} V} \operatorname{Tr}\left\{\mathcal{H}\left[\left[r_{\alpha}, \mathcal{P}\right],\left[r_{\beta}, \mathcal{P}\right]\right]\right\} .
$$

One further aesthetical improvement is desirable. We observe that any bulk observable (except the energy itself) must be invariant by translation of the energy zero, and hence must be a function of $\mathcal{H}-\mu$; and, in fact, $\mathcal{P}$ can be formally written as $\mathcal{P}=\theta(\mu-\mathcal{H})$, where $\theta$ is the step function. We, therefore, rewrite

$$
I_{\alpha \beta}=-\frac{i \pi e^{2}}{2 \hbar^{2} V} \operatorname{Tr}\left\{(\mathcal{H}-\mu)\left[\left[r_{\alpha}, \mathcal{P}\right],\left[r_{\beta}, \mathcal{P}\right]\right]\right\} .
$$

\section{Products of lattice-periodical operators}

We start with a simple lemma: if $f(\mathbf{r})$ is a lattice-periodical function, then its Fourier transform is

$$
\int e^{i \mathbf{k} \cdot \mathbf{r}} f(\mathbf{r})=\frac{(2 \pi)^{d}}{V_{\text {cell }}} \delta(\mathbf{k}) \int_{\text {cell }} d \mathbf{r} f(\mathbf{r}) .
$$

Any two lattice periodical operators can be written in Schrödinger representation as

$$
\begin{aligned}
\left\langle\mathbf{r}|\mathcal{A}| \mathbf{r}^{\prime}\right\rangle & =V_{\text {cell }} \int_{\mathrm{BZ}} \frac{d \mathbf{k}}{(2 \pi)^{d}} e^{i \mathbf{k} \cdot\left(\mathbf{r}-\mathbf{r}^{\prime}\right)}\left\langle\mathbf{r}\left|\mathcal{A}_{\mathbf{k}}\right| \mathbf{r}^{\prime}\right\rangle \\
\mathcal{A}_{k} & =\sum_{j j^{\prime}}\left|u_{j \mathbf{k}}\right\rangle\left\langle\psi_{j \mathbf{k}}|\mathcal{A}| \psi_{j^{\prime} \mathbf{k}}\right\rangle\left\langle u_{j^{\prime} \mathbf{k}}\right| . \\
\left\langle\mathbf{r}|\mathcal{B}| \mathbf{r}^{\prime}\right\rangle & =V_{\text {cell }} \int_{\mathrm{BZ}} \frac{d \mathbf{k}}{(2 \pi)^{d}} e^{i \mathbf{k} \cdot\left(\mathbf{r}-\mathbf{r}^{\prime}\right)}\left\langle\mathbf{r}\left|\mathcal{B}_{\mathbf{k}}\right| \mathbf{r}^{\prime}\right\rangle \\
\mathcal{B}_{k} & =\sum_{j j^{\prime}}\left|u_{j \mathbf{k}}\right\rangle\left\langle\psi_{j \mathbf{k}}|\mathcal{B}| \psi_{j^{\prime} \mathbf{k}}\right\rangle\left\langle u_{j^{\prime} \mathbf{k}}\right| .
\end{aligned}
$$

Their products obtain as

$$
\begin{array}{r}
\left\langle\mathbf{r}|\mathcal{A B}| \mathbf{r}^{\prime}\right\rangle=\int d \mathbf{r}^{\prime \prime}\left\langle\mathbf{r}|\mathcal{A}| \mathbf{r}^{\prime \prime}\right\rangle\left\langle\mathbf{r}^{\prime \prime}|\mathcal{B}| \mathbf{r}^{\prime}\right\rangle, \\
\left\langle\mathbf{r}\left|\mathcal{A}_{\mathbf{k}} \mathcal{B}_{\mathbf{k}^{\prime}}\right| \mathbf{r}^{\prime}\right\rangle=\int_{\text {cell }} d \mathbf{r}^{\prime \prime}\left\langle\mathbf{r}\left|\mathcal{A}_{\mathbf{k}}\right| \mathbf{r}^{\prime \prime}\right\rangle\left\langle\mathbf{r}^{\prime \prime}\left|\mathcal{B}_{\mathbf{k}^{\prime}}\right| \mathbf{r}^{\prime}\right\rangle .
\end{array}
$$

Notice that the integral in $d \mathbf{r}^{\prime \prime}$ is over $\mathbb{R}^{d}$ in the case of $\mathcal{A B}$, and over the crystal cell in the case of $\mathcal{A}_{\mathbf{k}} \mathcal{B}_{\mathbf{k}^{\prime}}$; notice also that $\mathcal{A}_{\mathbf{k}}$ and $\mathcal{B}_{\mathbf{k}^{\prime}}$ are lattice periodical in $\mathbf{r}$ and $\mathbf{r}^{\prime}$ independently.

We now address the diagonal term $\langle\mathbf{r}|\mathcal{A B}| \mathbf{r}\rangle$ : a latticeperiodical function. Its expression is

$$
\begin{aligned}
\langle\mathbf{r}|\mathcal{A B}| \mathbf{r}\rangle= & V_{\text {cell }}^{2} \int_{\mathrm{BZ}} \frac{d \mathbf{k}}{(2 \pi)^{d}} \int \frac{d \mathbf{k}^{\prime}}{(2 \pi)^{d}} e^{i\left(\mathbf{k}-\mathbf{k}^{\prime}\right) \cdot \mathbf{r}} \\
& \times \int d \mathbf{r}^{\prime \prime} e^{i\left(\mathbf{k}^{\prime}-\mathbf{k}\right) \cdot \mathbf{r}^{\prime \prime}}\left\langle\mathbf{r}\left|\mathcal{A}_{\mathbf{k}}\right| \mathbf{r}^{\prime \prime}\right\rangle\left\langle\mathbf{r}^{\prime \prime}\left|\mathcal{B}_{\mathbf{k}^{\prime}}\right| \mathbf{r}\right\rangle .
\end{aligned}
$$


By exploiting the above lemma, in the last line, one gets

$$
\begin{aligned}
\langle\mathbf{r}|\mathcal{A B}| \mathbf{r}\rangle= & V_{\text {cell }}^{2} \int_{\mathrm{BZ}} \frac{d \mathbf{k}}{(2 \pi)^{d}} \int \frac{d \mathbf{k}^{\prime}}{(2 \pi)^{d}} e^{i\left(\mathbf{k}-\mathbf{k}^{\prime}\right) \cdot \mathbf{r}} \\
& \times \frac{(2 \pi)^{d}}{V_{\text {cell }}} \delta\left(\mathbf{k}^{\prime}-\mathbf{k}\right) \int_{\text {cell }} d \mathbf{r}^{\prime \prime}\left\langle\mathbf{r}\left|\mathcal{A}_{\mathbf{k}}\right| \mathbf{r}^{\prime \prime}\right\rangle\left\langle\mathbf{r}^{\prime \prime}\left|\mathcal{B}_{\mathbf{k}^{\prime}}\right| \mathbf{r}\right\rangle \\
= & V_{\text {cell }} \int_{\mathrm{BZ}} \frac{d \mathbf{k}}{(2 \pi)^{d}}\left\langle\mathbf{r}\left|\mathcal{A}_{\mathbf{k}} \mathcal{B}_{\mathbf{k}}\right| \mathbf{r}\right\rangle .
\end{aligned}
$$

This process is associative and can be extended to the product of three operators:

$$
\langle\mathbf{r}|\mathcal{A B C}| \mathbf{r}\rangle=V_{\text {cell }} \int_{\mathrm{BZ}} \frac{d \mathbf{k}}{(2 \pi)^{d}}\left\langle\mathbf{r}\left|\mathcal{A}_{\mathbf{k}} \mathcal{B}_{\mathbf{k}} \mathcal{C}_{\mathbf{k}}\right| \mathbf{r}\right\rangle .
$$

In the main text, we identify $\mathcal{A}, \mathcal{B}$, and $\mathcal{C}$ with the lattice-periodical Hermitian operators $(\mathcal{H}-\mu), i\left[r_{\alpha}, \mathcal{P}\right]$, and $i\left[r_{\beta}, \mathcal{P}\right]$, respectively. Correspondingly, they enter the Fermi volume integral as $\left(\mathcal{H}_{\mathbf{k}}-\mu\right),-\partial_{k_{\alpha}} \mathcal{P}_{\mathbf{k}}$, and $-\partial_{k_{\beta}} \mathcal{P}_{\mathbf{k}}$.
[1] B. T. Thole, P. Carra, F. Sette, and G. van der Laan, Phys. Rev. Lett. 68, 1943 (1992).

[2] J. Kunes and P. M. Oppeneer, Phys. Rev. B 61, 15774 (2000).

[3] D. Ceresoli, T. Thonhauser, D. Vanderbilt, and R. Resta, Phys. Rev. B 74, 024408 (2006).

[4] D. Vanderbilt, Berry Phases in Electronic Structure Theory (Cambridge University Press, Cambridge, 2018).

[5] R. Bianco and R. Resta, Phys. Rev. Lett. 110, 087202 (2013).

[6] A. Marrazzo and R. Resta, Phys. Rev. Lett. 116, 137201 (2016).

[7] I. Souza and D. Vanderbilt, Phys. Rev. B 77, 054438 (2008).

[8] W. Kohn, Phys. Rev. Lett. 76, 3168 (1996).

[9] R. Resta, Phys. Rev. Lett. 80, 1800 (1998).

[10] When the Chern number is nonzero, the smooth Bloch orbitals cannot obey the so-called periodic gauge [4]; conversely, if chosen as periodical in $\mathbf{k}$, they cannot be smooth.

[11] D. J. Griffiths, Introduction to Electrodynamics, 3rd ed. (Prentice-Hall, New Jersey, 1999).
[12] R. Resta, arXiv:1703.00712.

[13] R. Bianco and R. Resta, Phys. Rev. B 93, 174417 (2016).

[14] R. Bianco and R. Resta, Phys. Rev. B 84, 241106(R) (2011).

[15] A. Marrazzo and R. Resta, Phys. Rev. B 95, 121114(R) (2017).

[16] A. Marrazzo and R. Resta, Phys. Rev. Lett. 122, 166602 (2019).

[17] R. Wu, D. Wang, and A. J. Freeman, Phys. Rev. Lett. 71, 3581 (1993).

[18] R. Benoist, P. Carra, and O. K. Andersen, Eur. Phys. J. B 18, 193 (2000).

[19] W. Kohn, Phys. Rev. 133, A171 (1964).

[20] D. Ceresoli, U. Gerstmann, A. P. Seitsonen, and F. Mauri, Phys. Rev. B 81, 060409(R) (2010).

[21] J. P. Hanke, F. Freimuth, A. K. Nandy, H. Zhang, S. Blugel, and Y. Mokrousov, Phys. Rev. B 94, 121114(R) (2016), and references quoted therein. 